TITLE:

\title{
Control of the spin polarization of photoelectrons/photoions using short laser pulses
}

AUTHOR(S):

Nakajima, T

CITATION:

Nakajima, T. Control of the spin polarization of

photoelectrons/photoions using short laser pulses. APPLIED PHYSICS LETTERS 2004, 84(19): 3786-3788

ISSUE DATE:

2004-05-10

URL:

http://hdl.handle.net/2433/50408

\section{RIGHT:}

Copyright 2004 American Institute of Physics. This article may be downloaded for personal use only. Any other use requires prior permission of the author and the American Institute of Physics. 


\title{
Control of the spin polarization of photoelectrons/photoions using short laser pulses
}

\author{
Takashi Nakajima ${ }^{\text {a) }}$ \\ Institute of Advanced Energy, Kyoto University, Gokasho, Uji, Kyoto 611-0011, Japan
}

(Received 9 December 2003; accepted 16 March 2004; published online 29 April 2004)

\begin{abstract}
We present a generic pump-probe scheme to control spin polarization of photoelectrons/photoions by short laser pulses. By coherently exciting fine structure manifolds of a multi-valence-electron system by the pump laser, a superposition of fine structure states is created. Since each fine structure state can be further decomposed into a superposition of various spin states of valence electrons, each spin component evolves differently in time. This means that varying the time delay between the pump and probe lasers leads to the control of spin states. Specific theoretical results are presented for two-valence-electron atoms, in particular for $\mathrm{Mg}$, which demonstrate that not only the degree of spin polarization but also its sign can be manipulated through time delay. Since the underline physics is rather general and transparent, the presented idea may be potentially applied to nanostructures such as quantum wells and quantum dots. (c) 2004 American Institute of Physics. [DOI: $10.1063 / 1.1739281]$
\end{abstract}

Although production, detection, and utilization of polarized electrons has been extensively studied in the past, ${ }^{1}$ recent theoretical and experimental progress on coherent control enables us to manipulate various properties of matters with lasers. Obviously controlling the spin degree of freedom is one of the most important subjects in Spintronics ${ }^{2}$ for decreasing electric power consumption, increasing data processing speed and integration densities. For the successful application of the spin degree of freedom into the conventional technology, we must establish efficient methods of manipulation, injection, and transport of spin-polarized electrons. If injection of spin currents can be done through ultrashort optical pulses, we would obtain another degree of freedom.

In a series of recent articles, ${ }^{3-6}$ we have investigated the production of spin-polarized electrons through photoionization of atoms. What we have found is that a proper choice of the levels and polarization of nanosecond lasers for excitation and ionization leads to the production of spin-polarized photoelectrons in atomic systems such as Xe and Sr. In particular, we have successfully demonstrated the simultaneous production of $64 \% \pm 9 \%$ spin-polarized photoions/ photoelectrons of $\mathrm{Sr}$ atoms ${ }^{6}$ through a simple scheme, which could open up a way toward the construction of a spinpolarized dual ion/electron source.

In the course of the work mentioned above, we came across an idea of using short laser pulses for the production of spin-polarized photoelectrons. In this letter, we explore the possibility of controlling the spin polarization of photoelectrons/photoions using short-pulse lasers. Our scheme is based on a pump-probe method. Briefly, by coherently exciting fine structure manifolds of a multi-valenceelectron system by the short pump laser, a superposition of fine structure states is created which oscillates in time after the pump pulse. Needless to say, its oscillation period, or

${ }^{a)}$ Electronic mail: t-nakajima@iae.kyoto-u.ac.jp state-flipping time between them, is determined by the fine structure energy splitting. Since each fine structure state can be further decomposed into a superposition of various spin states of valence electrons, we may be able to control the spin degree of freedom of photoelectrons simply by changing the time delay between the pump and probe lasers. Although we present specific theoretical results for one of the two-valence-electron atoms, $\mathrm{Mg}$, quite similar results can be expected for other two-valence-electron atoms such as $\mathrm{Ca}$, $\mathrm{Sr}$, and $\mathrm{Ba}$. Furthermore, we would like to emphasize that the physical picture presented in this letter is rather general in that it is essentially spin-orbit interaction of the system that plays an important role in controlling the spin degree of freedom. Therefore, the idea may be potentially applied to other systems such as semiconductors, quantum wells, and quantum dots: ${ }^{7}$ Although those systems may not have spherically symmetric potentials, the selection rule for dipole transition allows us to excite the particular magnetic sublevels if the quantization axis is properly chosen. Therefore we consider that at least a similar argument would hold for them.

Related to our work, by utilizing the quantum mechanical interference between one- and two-photon absorption together with the appropriate choice of beam polarization, optical control of spin currents has been demonstrated in semiconductors. ${ }^{8,9}$ In contrast, we coherently excite fine structure of a system (atom) by the pump laser, and let it evolve in time through spin-orbit interactions, and ionize after some delay by the probe laser so that the ionization takes place at the instant when the coherent states are in the desired spin states. Note that the two-path interference is not used here. Another related work is an application of pumpprobe scheme for quantum dots, in which the use of picosecond pulses has been proved to be very effective for the optical manipulation of the excitonic wave function, although spin was not involved in their work. ${ }^{7}$ Coherent nonlinear optical spectroscopy has also been demonstrated in GaAs quantum dots, showing similarities with atoms in the coherent optical interaction. ${ }^{10}$ 


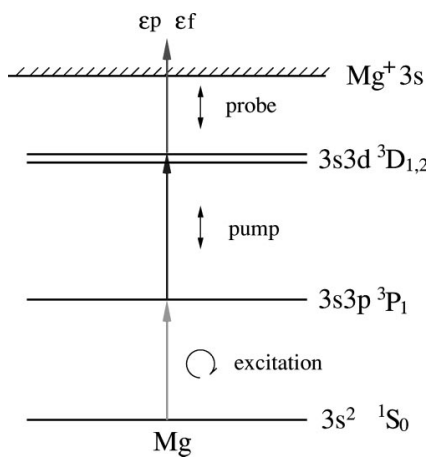

FIG. 1. Level scheme.

We should also note that a similar idea has been reported for a single-valence-electron atom, $\mathrm{K}:{ }^{11}$ A coherent superposition of $4^{2} P_{1 / 2}$ and $4^{2} P_{3 / 2}$ is created by the $150 \mathrm{fs}$ pump pulse. After a certain time delay, a probe pulse is fired for ionization. Spin polarization has been shown to modulate between $0 \%$ and $100 \%$ as a function of time delay. The essential difference between the single-valence-electron system and a two-valence-electron system presented here is that, for a two-valence-electron system it is not only photoelectrons but also photoions that can be polarized. ${ }^{6}$ Furthermore, as we show in this letter, it turned out that, for a multi-valenceelectron system at a certain energy region in the continuum, we can control not only the degree of spin polarization but also its sign simply by changing the time delay between the pump and probe pulses. We should emphasize that this feature is absent for the single-valence-electron system.

The level scheme we consider is shown in Fig. 1. We excite $\mathrm{Mg}$ atoms in the ground $3 s^{2}{ }^{1} S_{0}$ state to the triplet $3 s 3 p^{3} P_{1}(M=+1)$ state by a right-circularly polarized pulsed laser. Here $M$ denotes the projection of the total angular momentum $J$ onto the quantization axis. This excitation laser may have a long pulse duration. Once $3 s 3 p{ }^{3} P_{1}(M=+1)$ is populated, we do the pump-probe ionization by linearly polarized short-pulse lasers whose duration should be shorter than the fine structure coupling time. After the pump pulse with duration $\tau_{\text {pump }}$ to excite $3 s 3 d^{3} D_{1,2}$, the upper state wave function $|\Psi(t)\rangle$ is a superposition of $3 s 3 d^{3} D_{1}$ and ${ }^{3} D_{2}$, i.e.

$$
|\Psi(t)\rangle=\frac{-1}{2 \sqrt{6}}\left|3 s 3 d^{3} D_{1}\right\rangle+\frac{\sqrt{3}}{2 \sqrt{10}}\left|3 s 3 d^{3} D_{2}\right\rangle e^{-i \Delta E t},
$$

where the origin of the time $t$ is chosen to be the instant of the pump pulse, and $\Delta E$ is an energy difference between $3 s 3 d^{3} D_{1}$ and ${ }^{3} D_{2}$. Naturally it is required that $\tau_{\text {pump }}$ $\ll \Delta E^{-1}$. Note that $3 s 3 d^{3} D_{3}$ is not involved for excitation since such transition is dipole forbidden. For this specific example of $\mathrm{Mg}$, the pulse durations of the pump and probe pulses can be sub ns, since the fine structure splitting of $3 s 3 d^{3} D_{1,2}$ is $0.029 \mathrm{~cm}^{-1}$ corresponding to the $1.1 \mathrm{~ns}$ fine structure coupling time. For heavier two-valence-electron atoms, the coupling time may be shorter; 9 ps for $\mathrm{Ca}$ $4 s 4 d^{3} D_{1,2}$ and 2.2 ps for $\operatorname{Sr} 5 s 5 d^{3} D_{1,2}$, etc.

After a certain delay time, the probe pulse is fired to ionize atoms in the $3 s 3 d^{3} D_{1,2}$ states. Therefore, the Downloaded 09 Mar 2008 to 130.54.110.22. Redistribution subject photoelectron/photoion yields with spin-up/-down are written as

$$
P_{\sigma}(t)=\sum_{\ell} \int d \Omega\left|\left\langle 3 s k \ell, \sigma\left|D_{q}\right| \Psi(t)\right\rangle\right|^{2} \quad(\sigma=\uparrow, \downarrow),
$$

where $\int d \Omega$ is an angle integration and $\sigma=\uparrow, \downarrow$ denotes the spin state of photoelectrons/photoions. The term $k$ is the wave vector of the photoelectron and the subscript $q$ in $D_{q}$ stands for the polarization of the probe laser, i.e., $q=0, \pm 1$ for the linearly polarized, and right- and left-circularly polarized probe laser, respectively. Note that, as we have shown before, ${ }^{4}$ the degrees of spin polarization of photoelectrons and photoions are the same as long as the LS-coupling scheme is valid. After some angular momentum algebra, we obtain

$$
\begin{aligned}
P_{\sigma}(t)= & \sum_{\ell} \int d \Omega \mid \frac{-1}{2 \sqrt{6}}\left\langle 3 s k \ell, \sigma\left|D_{0}\right| 3 s 3 d^{3} D_{1}(M=+1)\right\rangle \\
& +\frac{\sqrt{3}}{2 \sqrt{10}}\left\langle 3 s k \ell, \sigma\left|D_{0}\right| 3 s 3 d^{3} D_{2}(M=+1)\right\rangle \\
& \times\left. e^{-i \Delta E t}\right|^{2} \quad(\sigma=\uparrow, \downarrow) .
\end{aligned}
$$

Since spin-orbit interaction is weak for $\mathrm{Mg}$, we may reasonably assume that the bound-free radial matrix elements from $3 s 3 d$ to $3 s k \ell$ do not depend on the total angular momentum, $J$, i.e., $R_{3 d}^{k \ell} \equiv R_{3 d 1}^{k \ell}=R_{3 d 2}^{k \ell}(\ell=p$ or $f)$. Then, we finally arrive at the following expressions:

$$
\begin{aligned}
P_{\uparrow}(t)= & \frac{49}{3600}\left(1+\frac{33}{49} \cos \Delta E t\right)\left|R_{3 d}^{k p}\right|^{2} \\
& +\frac{19}{1400}\left(1+\frac{13}{19} \cos \Delta E t\right)\left|R_{3 d}^{k f}\right|^{2}, \\
P_{\downarrow}(t)= & \frac{1}{400}(1+\cos \Delta E t)\left|R_{3 d}^{k p}\right|^{2} \\
& +\frac{1}{100}\left(1-\frac{3}{7} \cos \Delta E t\right)\left|R_{3 d}^{k f}\right|^{2} .
\end{aligned}
$$

Total photoelectron yield, $P_{\text {tot }}$, and the degree of spin polarization of photoelectrons/photoions, $P$, can be obtained from the relations $P_{\text {tot }}=P_{\uparrow}+P_{\downarrow}$ and $P=\left(P_{\uparrow}-P_{\downarrow}\right) /\left(P_{\uparrow}+P_{\downarrow}\right)$. From Eqs. (4) and (5) together with the definition of $P_{\text {tot }}$ and $P$, it is clear that not only the total photoelectron yield but also the degree of spin polarization are functions of time delay, and depend on the bound-free radial matrix elements, $R_{3 d}^{k p}$ and $R_{3 d}^{k f}$. Thus, for accurate estimation of these quantities, it is essential to know the accurate values of bound-free radial matrix elements, $R_{3 d}^{k p}$ and $R_{3 d}^{k f}$. We have calculated these quantities using a discretized basis set expanded using B-spline with two active electrons constructed on a frozen Hartree-Fock core. ${ }^{12}$ Starting from the $3 s 3 d^{3} D$ states, we have found two eminent autoionizing resonances, $3 p 3 d^{3} P$ and $3 p 3 d^{3} F$, located at the continuum energies of 2.7 and $2.3 \mathrm{eV}$, respectively. The $3 s 3 d^{3} \mathrm{D}$ atoms excited into the vicinity of $3 p 3 d^{3} P\left(3 p 3 d^{3} F\right)$ resonance by the probe laser eventually autoionize into the $3 s k p^{3} P\left(3 s k f^{3} F\right)$ cono AIP license or copyright; see http://apl.aip.org/apl/copyright.jsp 

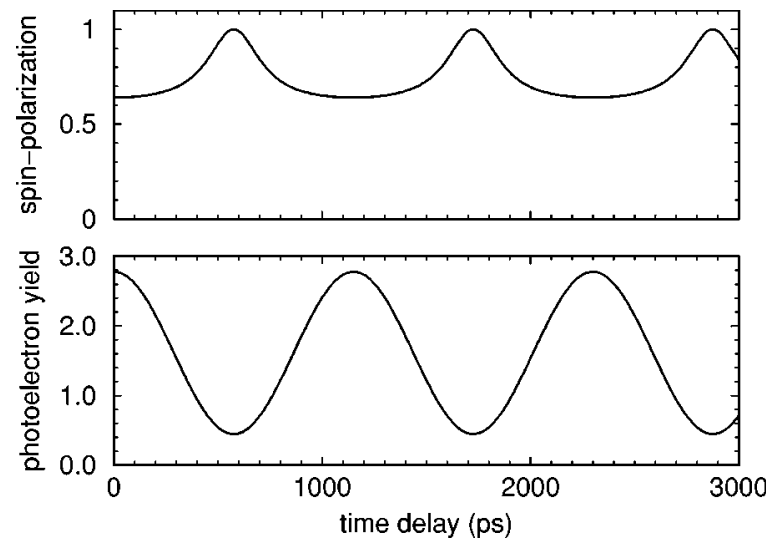

FIG. 2. Variation of spin polarization and total photoelectron yield as a function of time delay for $\left|R_{3 d}^{k p}\right| \gg\left|R_{3 d}^{k f}\right|$.

tinuum. This means that, at the continuum energy of $2.7 \mathrm{eV}$ $(2.3 \mathrm{eV}),\left|R_{3 d}^{k p}\right| \gg\left|R_{3 d}^{k f}\right|\left(\left|R_{3 d}^{k p}\right| \ll\left|R_{3 d}^{k f}\right|\right)$. Hence it is rather easy to experimentally realize both extremes, i.e., $\left|R_{3 d}^{k p}\right|$ $\gg\left|R_{3 d}^{k f}\right|$ and $\left|R_{3 d}^{k p}\right| \ll\left|R_{3 d}^{k f}\right|$, by changing the probe laser wavelength.

In Fig. 2, we show the variation of the total photoelectron yield and the degree of spin polarization as a function of delay time for $\left|R_{3 d}^{k p}\right| \gg\left|R_{3 d}^{k f}\right|$. The spin polarization modulates between 0.63 and 1. In Fig. 3 we show the result for the other extreme, $\left|R_{3 d}^{k p}\right| \ll\left|R_{3 d}^{k f}\right|$, where the behavior of the system is quite different. For this case the spin polarization oscillates between positive and negative values, 0.6 and -0.6 , as a function of delay time. This is the most interesting result of this work. Note that this behavior is peculiar to a multivalence-electron system, and cannot be seen for a singlevalence electron system: For a single-valence electron system the spin polarization oscillates between 0 and 1 , at best, as a function of delay time. It should be clear by now that the temporal behavior of the spin polarization through a pumpprobe method is quite different between a single-valenceelectron system and a two-valence-electron system.

In summary, we have presented a generic pump-probe scheme for controlling spin polarization of photoelectrons of a two-valence-electron system. It is essential that fine structure manifolds are coherently excited by a sufficiently short pump pulse. After the pump pulse, the coherently superposed fine structure states, which can be further decomposed into a superposition of different spin states of valence electrons, evolve differently in time. Therefore, spin polarization of photoelectrons modulates as a function of time delay between the pump and probe pulses. Since the underlying

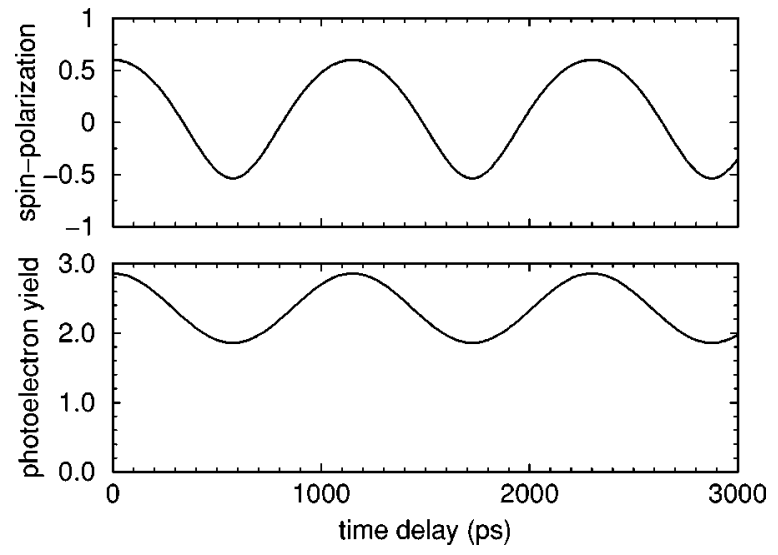

FIG. 3. Variation of spin polarization and total photoelectron yield as a function of time delay for $\left|R_{3 d}^{k p}\right| \ll\left|R_{3 d}^{k f}\right|$.

physics presented here is rather general and transparent, the idea may be potentially applied to other systems such as semiconductors, quantum wells, and quantum dots. ${ }^{7}$

Before closing this letter, we would like to point out that our scheme may also be suitable to create spin-entangled particles $^{13}$ if the photon energy of the probe laser is chosen so as to induce an excitation to a Rydberg state instead of a continuum state: If the field ionization is employed for ionization, spin entanglement generated by the pump laser will be destroyed by neither probe laser nor field ionization. Based on the theoretical results presented in this letter, experimental study is in course of preparation.

This work was supported by the Grant-in-Aid for scientific research from the Ministry of Education and Science of Japan.

${ }^{1}$ J. Kessler, Polarized Electrons, 2nd Ed. (Springer, Berlin, 1985).

${ }^{2}$ S. A. Wolf, D. D. Awschalom, R. A. Buhrman, J. M. Daughton, S. von Molnár, M. L. Roukes, A. Y. Chtchelkanova, and D. M. Treger, Science 294, 1488 (2001).

${ }^{3}$ T. Nakajima and P. Lambropoulos, Europhys. Lett. 57, 25 (2002).

${ }^{4}$ T. Nakajima and N. Yonekura, J. Chem. Phys. 117, 2112 (2002).

${ }^{5}$ T. Nakajima and L. A. A. Nikolopoulos, Phys. Rev. A 68, 013413 (2003).

${ }^{6}$ T. Nakajima, N. Yonekura, Y. Matsuo, T. Kobayashi, and Y. Fukuyama, Appl. Phys. Lett. 83, 2103 (2003).

${ }^{7}$ N. H. Bonadeo, J. Erland, D. Gammom, D. Park, D. S. Katzer, and D. G. Steel, Science 282, 1473 (1998).

${ }^{8}$ R. D. Bhat and J. E. Sipe, Phys. Rev. Lett. 85, 5432 (2000).

${ }^{9}$ J. Hübner, W. W. Rühle, M. Klude, D. Hommel, R. D. Bhat, J. E. Sipe, and H. M. van Driel, Phys. Rev. Lett. 90, 216601 (2003).

${ }^{10}$ N. H. Bonadeo, G. Chen, D. Gammon, D. S. Katzer, D. Park, and D. G. Steel, Phys. Rev. Lett. 81, 2759 (1998).

${ }^{11}$ M. A. Bouchene, S. Zamith, and B. Girard, J. Phys. B 34, 1497 (2001).

${ }^{12}$ T. K. Fang and T. N. Chang, Phys. Rev. A 61, 052716 (2000).

${ }^{13}$ S. K. Sekatskiǔ, JETP Lett. 78, 405 (2003). 